Math. Model. Nat. Phenom.

Vol. 9, No. 4, 2014, pp. 20-37

DOI: $10.1051 / \mathrm{mmnp} / 20149403$

\title{
Constant Versus Periodic Fishing: Age Structured Optimal Control Approach
}

\author{
A. O. Belyakov ${ }^{1,2} *$, V. M. Veliov ${ }^{1}$ \\ ${ }^{1}$ ORCOS, Vienna University of Technology \\ ${ }^{2}$ Institute of Mechanics, Lomonosov Moscow State University
}

\begin{abstract}
The paper investigates an age-structured infinite-horizon optimal control model of harvesting a biological resource, interpreted as fish. Time and age are considered as continuum variables. The main result shows that in case of selective fishing, where only fish of prescribed sizes is harvested, it may be advantageous in the log run to implement a periodic fishing effort, rather than constant (the latter suggested by single-fish models that disregard the age-heterogeneity). Thus taking into account the age-structure of the fish may qualitatively change the theoretically optimal fishing mode. This result is obtained by developing a technique for reliable numerical verification of second order necessary optimality conditions for the considered problem. This technique could be useful for other optimal control problems of periodic age-structured systems.
\end{abstract}

Keywords and phrases: age-structured systems, periodic control, optimal fishing, properness test

Mathematics Subject Classification: 49N20, 35F46

\section{Introduction}

The main goal of this paper is to shed some more light on the following question: is a constant fishing effort in a single fish population optimal in terms of the net revenue in the long run, or is a periodic fishing better? It is well known that using standard ODE optimization models, where the fish is homogeneous, the optimal harvesting effort is constant in the long run (see e.g. [6, Chapter 4]). In this paper we show that if the age-structure of the fish population is taken into account, then a periodic harvesting may become more profitable. For this effect to occur, it is essential to consider selective harvesting, where only fish in a specified size-range is harvested. Apart from this selectivity (which can be implemented by technical tools) the fishing effort is considered as depending only on time, in contrast to forestry models, where harvesting can have a more detailed size-structure.

The analysis presented in this paper is based on an averaged infinite-time horizon optimal control problem for an age-structured PDE system, where the time-dependent fishing effort is viewed as a control.

Modeling in fishery economics and ecology has a long history, see e.g. the excellent survey [13], from which we quote the following:

${ }^{*}$ Corresponding author. E-mail: anton.belyakov@tuwien.ac.at 
"Aside from the fact that understanding optimal harvesting of age-structured fish populations is a theoretically fascinating open question, there is no doubt that the pressure to include the age-structure in economically relevant fishery models is steadily increasing."

We partly fulfill this gap in the existing literature. The model we consider is continuous in age and time and has a similar first order PDE form as the one considered in [3,4]. However, the model in [3,4] assumes fishing in all ages (sizes), while for superiority of periodic fishing (in comparison with asymptotically constant one) it is of key importance that the fishing is selective: say, only fish of prescribed sizes is harvested, as it happens in practice. The results in $[3,4]$ are not applicable in this case.

This paper takes the averaged net revenue of fishing in the long run as a performance criterion to be maximized. Alternatively, one can consider the usual maximization of the total discounted net revenue, which is also economically meaningful. The technique presented below may be useful also for this problem.

Our approach consists of the following steps. First we characterize the optimal constant fishing effort. Second, using ideas from [7], we develop a procedure (called in [7] properness test) for checking if the optimal constant control dominates all its additive (small) periodic variations of sinusoidal type. This properness test is based on second order variational analysis of the problem at hand. A remarkable feature is that it requires only solving ODE systems which are easily constructed from the original problems data, and are independent from the size of the variation. Third, for particular data specifications we perform the test numerically in a reliable way, which is possible, thanks to the abovementioned feature of the test. Cases where proper periodic fishing strictly dominates the optimal constant fishing are presented.

On the other hand, if the fishing is not selective the picture changes. We prove that optimal fishing which is constant always exists in this case. This shows that the selectivity of fishing is the reason for which non-constant fishing efforts may be more profitable than the constant ones.

We mention that the model considered in the next section, although presented in terms of fish, might be applicable also to other biological resources.

The paper is organized as follows. In the next section we present the model, the main assumptions, and a result from [1] that provides a base for the further analysis. In Section 3 we characterize the optimal constant fishing and outline a numerical approach for its calculation. Then in Section 4 we develop the technique of the properness test, and present some numerical results exhibiting cases where proper periodic fishing is strictly more profitable than the optimal constant one. Section 5 is devoted to the case of non-selective fishing, and Sections 6 contains some concluding remarks. The proof of an auxiliary proposition is given in the Appendix.

\section{The optimal fishing problem}

In the model below we consider a fish population in a closed natural basin. For the main issue investigated in this paper - constant versus periodic harvesting - it is substantial that the model takes into account the age-structure of the fish population. The variable indicating age is denoted by $a$, and it is assumed that $a \in[0, A]$, where $A$ is a proxy for the maximal age that a fish may achieve (meaning, that fishes of age higher than $A$ can be disregarded, since they are not fertile and their total biomass is negligible). Denote by $n(t, \cdot)$ the (non-normalized) age-density of fish at time $t \in[0,+\infty)$.

The main age-specific data involved in the model are the natural mortality rate, $\mu(a)$, the fertility rate, $\beta(a)$, and the fish size, $\gamma(a)$ (it is assumed that there is a one-to-one relation between age and size). Moreover, there is an additional mortality rate $M(z)$ depending on the total biomass, $z$. In addition, the fish is harvested with intensity $v(t)$. However, the harvesting is selective - too small fish may either be of no commercial value or their harvesting be prohibited by regulation. The selectivity is represented by a function $\chi(a)$ : the harvested flow of fish of age $a$ resulting from fishing effort $v(t)$ in a population with density $n(t, a)$ is $\chi(a) v(t) n(t, a)$. Typically, $\chi(a)$ is zero for small ages and equals 1 after a certain age. 
The dynamics of the fish population is described by the standard age-structured system

$$
\begin{aligned}
\left(\frac{\partial}{\partial t}+\frac{\partial}{\partial a}\right) n(t, a) & =-(\mu(a)+M(z(t))+v(t) \chi(a)) n(t, a), \quad(t, a) \in[0, \infty) \times[0, A], \\
n(0, a) & =n^{0}(a), \\
n(t, 0) & =\int_{0}^{A} \beta(a) n(t, a) \mathrm{d} a, \\
z(t) & =\int_{0}^{A} \gamma(a) n(t, a) \mathrm{d} a,
\end{aligned}
$$

where $n^{0}(a)$ is a given initial density, $n(t, 0)$ is the size of the newborn fish population, $z(t)$ is the total biomass at time $t$. We use the classical PDE representation of the transport-reaction equation (2.1), but the left-hand side should be interpreted as the directional derivative of $n$ in the direction $(1,1)$ :

$$
\left(\frac{\partial}{\partial t}+\frac{\partial}{\partial a}\right) n(t, a)=\mathcal{D} n(t, a):=\lim _{\varepsilon \rightarrow 0+} \frac{n(t+\varepsilon, a+\varepsilon)-n(t, a)}{\varepsilon}
$$

(further on we use the notation $\mathcal{D}$ for this derivative). Since $n$ is assumed absolutely continuous on almost every characteristic line $t-a=$ const., the traces $n(t, 0)$ and $n(0, a)$ make sense (see e.g. $[2,10,12,15]$ for the precise meaning of a solution of (2.1)-(2.4)).

Notice that the age-specific natural mortality $\mu(a)$ and the mortality due to competition, $M(z)$, enter additively in (2.1), therefore the model is separable, in the terminology of [12, Chapter V, Section 3].

The following assumptions are standing all over the paper.

Assumption (A1). The functions $\mu, \beta, \gamma, \chi:[0, A] \rightarrow[0, \infty)$ are measurable and bounded; $\beta(a) \geq \beta_{0}>0$ on some age-interval of length $\bar{T}>0 ; \gamma(a) \geq \gamma_{0}>0$ for all $a \in[0, A] ; \chi$ is not identically equal to zero. Assumption (A2). The following inequality holds:

$$
\int_{0}^{A} \beta(a) e^{-\int_{0}^{a} \mu(\eta) \mathrm{d} \eta} \mathrm{d} a>1 .
$$

Assumption (A3). $M:(-\infty,+\infty) \rightarrow(-\infty,+\infty)$ is an increasing, two times continuously differentiable function, $M(0)=0$, and $\lim _{z \rightarrow+\infty} M(z)=+\infty$.

Assumption (A4). There exists $t \in[0, A)$ such that $\operatorname{meas}\left\{a \in[t, A): \beta(a) n^{0}(a-t)>0\right\}>0$.

Assumption (A2) is known as above replacement fertility (if the initial data $n^{0}$ is non-trivial in the sense of (A4), and there is no harvesting, then the population grows as long as its density is low enough). Assumption (A3) ensures that the population cannot grow infinitely, (A4) is a necessary and sufficient condition for the initial population $n^{0}$ to produce a positive number of off-springs (see Remark 2.3.1 in $[2$, Chapter 2]).

It is well known that on the above assumptions for every measurable and bounded function $v$ a solution of system (2.1)-(2.4) exists and is unique (see e.g. [2, Theorem 2.1.1]).

We mention that the control system (2.1)-(2.4) is similar to the one considered in [3] with the substantial difference that here the harvesting rate $\chi(a) v(t)$ is age-dependent, while in [3] the mortality due to harvesting is independent of age. The last property is crucial for the analysis in [3], while the selectivity of fishing is a key feature in the present paper.

We consider the optimal control problem

$$
\begin{aligned}
J(v(\cdot)) & :=\liminf _{\tau \rightarrow \infty} \frac{1}{\tau} \int_{0}^{\tau}\left(\int_{0}^{A} \gamma(a) \chi(a) n(t, a) \mathrm{d} a-c\right) v(t) \mathrm{d} t \rightarrow \max _{v(\cdot)}, \\
v(t) & \in[0, V], \forall t \geq 0
\end{aligned}
$$


where $V$ is an upper bound for the fishing effort, $c v$ is the cost of fishing with effort $v$. That is, a fishing effort $v(t)$ is sought, which maximizes the average profit of fishing in the long run. Admissible controls in the above problem are all measurable functions $v:[0, \infty) \rightarrow[0, V]$.

We restate the main question studied in this paper-constant versus periodic fishing -in the following (somewhat weaker) way: is there a constant optimal control, or every constant control $v(t)=v$ can be strictly dominated (in the terms of the objective functional) by a proper periodic one. Contrary to what can be obtained by using homogeneous models (in which the age of the fish is disregarded and the optimal fishing effort is constant) the answer of this question turns out to depend on the particular data configuration.

We conclude this section with a result proved in [1], which plays a crucial role in the next two sections.

Theorem 2.1. ([1, Theorem 2.2]) If $v(\cdot)$ is continuous and T-periodic with $T \in(0, \bar{T}]$, then system (2.1)-(2.4) has a unique solution $n$ and

$$
\lim _{t \rightarrow \infty}\|\bar{n}(t, \cdot)-n(t, \cdot)\|_{L^{\infty}(0, A)}=0,
$$

where $\bar{n}$ is the maximal non-negative solution of the system

$$
\begin{aligned}
\mathcal{D} \bar{n}(t, a) & =-(\mu(a)+M(\bar{z}(t))+v(t) \chi(a)) \bar{n}(t, a), \quad(t, a) \in[0, T] \times[0, A), \\
\bar{n}(0, a) & =\bar{n}(T, a), \\
\bar{n}(t, 0) & =\int_{0}^{A} \beta(a) \bar{n}(t, a) \mathrm{d} a \\
\bar{z}(t) & =\int_{0}^{A} \gamma(a) \bar{n}(t, a) \mathrm{d} a .
\end{aligned}
$$

Remark 2.2. Our assumptions (A1)-(A4) are somewhat weaker than those in [1]. In particular, the function $\gamma(a) \equiv 1$ in [1], but our assumption $\gamma(a) \geq \gamma_{0}>0$ in (A1) allows to apply the same proof. The same applies to (A4), which guarantees that the non-harvesting population will not become extinct.

It has to be mentioned also that system (2.6)-(2.9) has at most two non-negative solutions, one of which is identically zero.

\section{Optimal constant fishing effort}

In this section we consider the optimal control problem (2.5), (2.1)-(2.4) in the set of constant admissible controls $v \in[0, V]$. As we argue below an optimal $v^{*} \in[0, V]$ does exist.

Let $v \in[0, V]$ be any constant control, and let $n(t, a)$ be the corresponding solution of $(2.1)-(2.4)$. We can apply Theorem 2.1 with any $T>0$. Equation (2.7) implies that $\bar{n}(t, a)=\bar{n}(a)$ is time-invariant. Then the limit system (2.6)-(2.9) reduces to

$$
\begin{aligned}
\bar{n}^{\prime}(a) & =-(\mu(a)+M(\bar{z})+v \chi(a)) \bar{n}(a), \quad a \in[0, A], \\
\bar{n}(0) & =\int_{0}^{A} \beta(a) \bar{n}(a) \mathrm{d} a, \\
\bar{z} & =\int_{0}^{A} \gamma(a) \bar{n}(a) \mathrm{d} a .
\end{aligned}
$$

Passing to the limit in (2.5) (which is possible, since $\gamma$ and $\chi$ are bounded) gives

$$
J(v)=v \int_{0}^{A} \gamma(a) \chi(a) \bar{n}(a) \mathrm{d} a-c v,
$$


where $\bar{n}$ is the maximal non-negative solution of (3.1)-(3.3). If $\bar{n}$ is not identically equal to zero then it is strictly positive for all $a \in[0, A]$.

In the next paragraphs we obtain a more explicit representation of the function $J$. Let us denote

$$
\bar{\alpha}:=M(\bar{z}), \quad \bar{b}:=\int_{0}^{A} \beta(a) \bar{n}(a) \mathrm{d} a .
$$

Then the solution $\bar{n}$ of (3.1), (3.2) takes the form

$$
\bar{n}(a)=\Pi(a ; v, \bar{\alpha}) \bar{b}
$$

where

$$
\Pi(a ; v, \alpha):=\exp \left(-\alpha a-\int_{0}^{a}(\mu(\eta)+v \chi(\eta)) \mathrm{d} \eta\right) .
$$

Substituting the above expression for $\bar{n}$ in (3.2) and (3.3) we obtain that $\alpha=\bar{\alpha}$ and $b=\bar{b}$ satisfy the equations

$$
b=b \int_{0}^{A} \beta(a) \Pi(a ; v, \alpha) \mathrm{d} a, \quad M^{-1}(\alpha)=b \int_{0}^{A} \gamma(a) \Pi(a ; v, \alpha) \mathrm{d} a .
$$

From the first equation we obtain that either $\bar{b}=0$ or $\bar{\alpha}$ satisfies the equation

$$
1=R(v, \alpha),
$$

where

$$
R(v, \alpha):=\int_{0}^{A} \beta(a) \Pi(a ; v, \alpha) \mathrm{d} a
$$

is the net reproduction rate for fixed $v$ and $\alpha$.

Notice that $R$ is a continuous function, it is monotone decreasing in $v$, and strictly monotone decreasing in $\alpha \geq 0$. Moreover, according to (A2)

$$
R(0,0)=\int_{0}^{A} \beta(a) e^{-\int_{0}^{a} \mu(\eta) \mathrm{d} \eta} \mathrm{d} a>1 .
$$

Since $\chi$ is not identically zero, there exists a (maximal) number $\bar{v}>0$ such that

$$
R(v, 0)>1 \text { for } v \in[0, \bar{v}), \quad \text { and } \quad R(v, 0) \leq 1 \quad \text { for } v \geq \bar{v} .
$$

Then taking into account the monotonicity of $R$ in $\alpha$ we obtain that for $v \geq \bar{v}$ equation (3.6) has no solution with $\alpha>0$, which implies due to the first equation in (3.5) that $\bar{b}=0$.

Since $R(v, \cdot)$ is strictly monotone and $R(v, \alpha) \rightarrow 0$ when $\alpha \rightarrow 0$, we have that for $v \in[0, \bar{v})$ equation (3.6) has a unique solution $\alpha=\alpha(v)>0$. In this case $\bar{b}$ can be determined from the second equation in (3.5). As a result, we obtain that for $v \in[0, \bar{v})$

$$
\bar{n}(a)=M^{-1}(\alpha(v)) P(v, \alpha(v)),
$$

where

$$
P(v, \alpha):=\frac{\Pi(a ; v, \alpha)}{\int_{0}^{A} \gamma(\theta) \Pi(\theta ; v, \alpha) \mathrm{d} \theta} .
$$

For $v \geq \bar{v}$ we have $\bar{b}=0$, hence $\bar{n}(a) \equiv 0$.

Combining the two cases we obtain the following representation of the objective function $J$ :

$$
J(v)=\left\{\begin{array}{cl}
\left(M^{-1}(\alpha(v)) P(v, \alpha(v))-c\right) v & \text { if } v \in[0, \bar{v}), \\
-c v & \text { if } v \in[\bar{v}, V] .
\end{array}\right.
$$


Thus the optimization problem (2.5), (2.1)-(2.4) in the set of all constant controls $v \in[0, V]$ is equivalent to

$$
\max _{v \in[0, V]} J(v)
$$

with $J$ now given by (3.7).

Proposition 3.1. The optimal control problem (2.5), (2.1)-(2.4) has a solution in the set of all constant controls $v \in[0, V]$.

Indeed, due to $\alpha(\bar{v})=0$ we have $M^{-1}(\alpha(\bar{v}))=0$, hence the function $J$ in (3.7) is continuous. Then an optimal solution $v^{*} \in[0, V]$ of problem (3.8) does exist, and it is a solution also of (2.5), (2.1)-(2.4) in the class of constant admissible controls.

Below we briefly describe a procedure for solving the problem with constant controls numerically. Let $v^{*}$ be a solution. Since $J(0)=0$ and $J(v)=-c v \leq 0$ for $v \geq \bar{v}$, we have that $v^{*} \in[0, \min \{\bar{v}, V\}]=:[0, \bar{V}]$. In this interval the function $J$ is differentiable (only from one side, if $v=0$ or $v=\bar{v}$ ), and the first order optimality condition that $v^{*}$ must satisfy reads as

$$
\begin{cases}J_{v}(v) \leq 0, & \text { if } v=0, \\ J_{v}(v)=0, & \text { if } v \in(0, \bar{V}), \\ J_{v}(v) \geq 0, & \text { if } v=\bar{V} .\end{cases}
$$

Thus in order to obtain numerically the solution $v^{*}$ we need to calculate the derivative $J_{v}$ for $v \in[0, \bar{V}]$.

From (3.6) we have

$$
\alpha_{v}(v)=-\frac{R_{v}(v, \alpha(v))}{R_{\alpha}(v, \alpha(v))}
$$

Then

$$
\begin{aligned}
J_{v}(v)= & M^{-1}(\alpha(v))\left(P(v, \alpha(v))+v P_{v}(v, \alpha(v))\right)-c \\
& -v\left(\left(M^{-1}\right)^{\prime}(\alpha(v)) P(v, \alpha(v))+M^{-1}(\alpha(v)) P_{\alpha}(v, \alpha(v))\right) \frac{R_{v}(v, \alpha(v))}{R_{\alpha}(v, \alpha(v))} .
\end{aligned}
$$

The calculation of the derivatives that appear in this formula is also straightforward. We give the formulae for completeness:

$$
\begin{gathered}
\Pi_{v}(a ; v, \alpha)=-\left(\int_{0}^{a} \chi(\eta) \mathrm{d} \eta\right) \Pi(a ; v, \alpha) \\
\Pi_{\alpha}(a ; v, \alpha)=-a \Pi(a ; v, \alpha) \\
R_{v}(v, \alpha)=\int_{0}^{A} \beta(a) \Pi_{v}(a ; v, \alpha) \mathrm{d} a \\
R_{\alpha}(v, \alpha)=\int_{0}^{A} \beta(a) \Pi_{\alpha}(a ; v, \alpha) \mathrm{d} a \\
P_{v}(v, \alpha)=\frac{\int_{0}^{A} \chi(a) \gamma(a) \Pi_{v}(a ; v, \alpha) \mathrm{d} a-P(v, \alpha) \int_{0}^{A} \gamma(a) \Pi_{v}(a ; v, \alpha) \mathrm{d} a}{\int_{0}^{A} \gamma(a) \Pi(a ; v, \alpha) \mathrm{d} a} \\
P_{\alpha}(v, \alpha)=\frac{\int_{0}^{A} \chi(a) \gamma(a) \Pi_{\alpha}(a ; v, \alpha) \mathrm{d} a-P(v, \alpha) \int_{0}^{A} \gamma(a) \Pi_{\alpha}(a ; v, \alpha) \mathrm{d} a}{\int_{0}^{A} \gamma(a) \Pi(a ; v, \alpha) \mathrm{d} a}
\end{gathered}
$$

Then any gradient method for scalar maximization can be utilized for computation of (an approximation of) the solution $v^{*}$. 

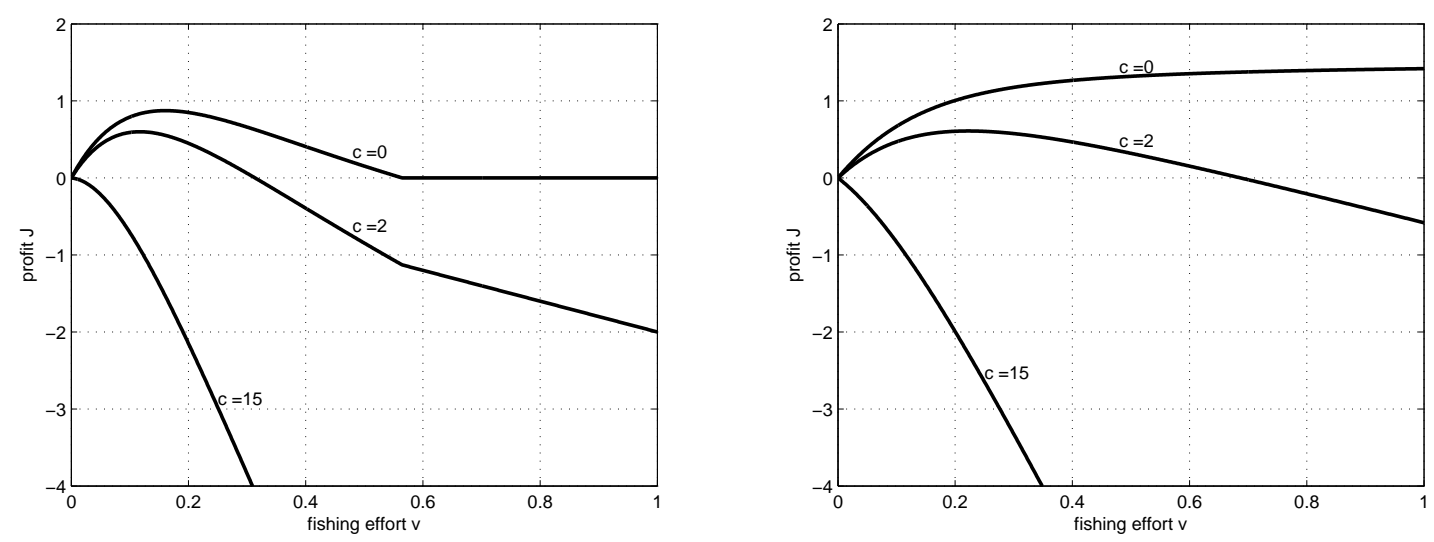

Figure 1. Objective functional $J(v)$ for different fishing costs $c$ and for two minimal fishing ages: 15 (left) and 20 (right) units of time.

Remark 3.2. Let us consider the possible appearance of a "boundary" optimal control $v^{*} \in\{0, \bar{V}\}$ from a practical point of view. We may exclude the possibility that $v^{*}=V$, since in the long run the capacity of the fishing facilities would be flexible enough to adapt to any profitable activity. In the case $v^{*}=\bar{v}$ we have $J\left(v^{*}\right)=-c v^{*} \leq 0$. Thus sustainable profitable harvesting is not possible in this case. Moreover, the case $v^{*}=0$ can appear only if the cost of harvesting $c$ is sufficiently large. Indeed, if $c=0$, assumptions (A2)-(A4) imply that with a sufficiently small constant harvesting effort $v>0$ the fish population will not become asymptotically extinct, hence $J(v)>0=J(0)$. If $c$ is sufficiently large, then $v^{*}=0$ will be optimal, however, sustainable profitable harvesting is not possible also in this case. At some places below we ignore these possibilities, focusing on the interesting case $v^{*} \in(0, \bar{V})$.

Figure 1 illustrates function $J(v)$ for three different values of the fishing cost $c$ (on the same plot), and two selection functions: $\chi(a)=\chi_{[15, A]}(a)$ and $\chi(a)=\chi_{[20, A]}(a), A=30$. In all cases the objective functional $J$ in (3.7) has a unique maximum in $[0, V]=[0,1]$. For $c=15$ no harvesting is optimal, while for $c=0$ harvesting at the upper bound $v=1$ is optimal for the second selection function $\chi$. In the other cases the optimal constant harvesting effort is in the interior of the admissible domain $[0,1]$.

\section{Can a proper periodic fishing be better than any constant one?}

We investigate this question in the following way. Let $v^{*} \in(0, \bar{V})$ be an optimal solution of problem (2.5), (2.1)-(2.4) in the set of all constant admissible controls (see the previous section and Remark 3.2). We ask:

Can the objective value $J\left(v^{*}\right)$ be improved by a proper periodic perturbation $v(t)=v^{*}+\varepsilon w(t) \in[0, \bar{V}]$ ?

Here $w$ is continuous and $T$-periodic with its minimal period $T \in(0, \bar{T}]$, (the number $\bar{T}$ is defined in Assumption (A1)) and $v(t) \in[0, \bar{V}]$ for all sufficiently small $\varepsilon>0$. Specifically, we take

$$
w(t)=\sin (\Omega t), \quad \Omega=\frac{2 \pi}{T}, \quad T \in(0, \bar{T}]
$$




\subsection{Theoretical ground}

Thanks to Theorem 2.1 and the boundedness of $\gamma, \chi$, and $v(\cdot)$, it is a routine task to represent the objective functional $J$ in (2.5) in the following way:

$$
J(T, v)=\frac{1}{T} \int_{0}^{T}\left(\int_{0}^{A} \gamma(a) \chi(a) \bar{n}(t, a) \mathrm{d} a-c\right) v(t) \mathrm{d} t,
$$

where now the function $v$ is restricted to $[0, T]$ and $\bar{n}$ (together with $\bar{z}$ ) is the corresponding maximal non-negative solution of (2.6)-(2.9).

We remind that the solution corresponding to $\varepsilon=0$, that is for $v(t)=v^{*}$, is time-invariant and is the maximal non-negative solution of system (3.1)-(3.3). Further we denote this solution by $\left(n^{*}(a), z^{*}\right)$. Due to $v^{*}>0$ and $v^{*}<\bar{V}$, the function $n^{*}$ is not identically zero, hence it is strictly positive for every $a \in[0, A]$.

In the sequel we shall follow the idea of the approach of properness test suggested in [7]. Namely, we consider $T$ and the function $w$ as fixed as above, and investigate the dependence on $\varepsilon$ of

$$
\hat{J}(\varepsilon):=J\left(T, v^{*}+\varepsilon w(\cdot)\right) .
$$

We shall prove that $\hat{J}$ has the following expansion at $\varepsilon=0$ :

$$
\hat{J}(\varepsilon)=J\left(T, v^{*}\right)+\varepsilon \hat{J}^{\prime}+\frac{\varepsilon^{2}}{2} \hat{J}^{\prime \prime}+o\left(\varepsilon^{2}\right),
$$

where $o\left(\varepsilon^{2}\right) / \varepsilon^{2} \rightarrow 0$ with $\varepsilon$ and $J^{\prime}$ and $J^{\prime \prime}$ are numbers to be specified (in fact, these are the first two derivatives of $\hat{J}$ at $\varepsilon=0$, although from what follows this is clear only for $J^{\prime}$ ). Moreover, we shall prove that, due to the structure of system (2.6)-(2.9) and the specific choice of $w$, it holds that $\hat{J}^{\prime}=0$. Then if for an appropriately chosen $T>0$ we have $\hat{J}^{\prime \prime}>0$, then $J\left(T, v^{*}\right)<J\left(T, v^{*}+\varepsilon w(\cdot)\right)$ for all sufficiently small $\varepsilon>0$. Thus the constant control $v^{*}$ is strictly dominated by a proper periodic one.

To work out all this, however, we need an additional assumption about system (2.6)-(2.9), which is known as strong metric regularity (see [8]). It involves a disturbed version of system (2.6):

$$
\mathcal{D} \bar{n}(t, a)=-\left(\mu(a)+M(z(t))+v^{*} \chi(a)\right) \bar{n}(t, a)+m(t, a), \quad(t, a) \in[0, T] \times[0, A],
$$

completed with $(2.7)-(2.9)$.

Assumption (H). There exist numbers $\varepsilon_{0}>0, \delta>0$, and $d$ such that for every two disturbances $m^{1}$ and $m^{2}$ which are measurable, bounded and $T$-periodic, and for which

$$
\left\|m^{i}\right\|_{\infty} \leq \varepsilon_{0}
$$

the disturbed problems (4.3), (2.7)-(2.9) (with $m^{1}$ and $m^{2}$ in (4.3)) have unique solutions, $n^{1}$ and $n^{2}$, in the $\delta$-neighborhood of $\bar{n}$ in the space $L_{\infty}([0, T] \times[0, A])$, and

$$
\left\|n^{1}-n^{2}\right\|_{\infty} \leq d\left\|m^{1}-m^{2}\right\|_{\infty} .
$$

Remark 4.1. We mention that although strong metric regularity is a fundamental property (essentially, due to its stability with respect to small Lipschitz perturbations) it seems not to be systematically investigated for periodic problems like (2.6)-(2.9). Such an investigation will be of interest from the view point of stability of the solution with respect to migration and (non-linear) perturbations, as well as for error analysis of numerical schemes (see e.g. $[5,9]$ ). However, it is not in the scope of the present paper. 
Let $\bar{n}_{\varepsilon}$ be the maximal non-negative solution of system (2.6)-(2.9) corresponding to the control function $v(t)=v^{*}+\varepsilon w(t)$. In the next paragraph we shall prove that $\bar{n}_{\varepsilon}$ can be represented as $\bar{n}_{\varepsilon}(t, a)=$ $\tilde{n}_{\varepsilon}(t, a)+o\left(\varepsilon^{2}\right)$, where $o\left(\varepsilon^{2}\right)=o\left(\varepsilon^{2} ; t, a\right)$ converges to zero faster than $\varepsilon^{2}$ when $\varepsilon \rightarrow 0$, uniformly in $(t, a) \in[0, T] \times[0, A]$,

$$
\tilde{n}_{\varepsilon}(t, a)=n^{*}(a)+\varepsilon n_{1}(t, a)+\frac{\varepsilon^{2}}{2} n_{2}(t, a),
$$

and the functions $n_{1}(t, a)$ and $n_{2}(t, a),(t, a) \in[0, T] \times[0, A]$, are measurable, bounded, absolutely continuous on every line $t-a=$ const., and $T$-periodic in $t$. The later functions and the corresponding integrals

$$
z_{i}(t)=\int_{0}^{A} \gamma(a) n_{i}(t, a) \mathrm{d} a, \quad i=1,2,
$$

will be obtained as follows.

We insert $v(t)=v^{*}+\varepsilon w(t)$, the expression for $\tilde{n}_{\varepsilon}$ in (4.4), and the expression

$$
\tilde{z}_{\varepsilon}(t):=z^{*}+\varepsilon z_{1}(t)+\frac{\varepsilon^{2}}{2} z_{2}(t)
$$

in equation $(2.6)$ (at the place of $(\bar{n}, \bar{z})$ ), where we also replace $M\left(\tilde{z}_{\varepsilon}(t)\right)$ with its linear-quadratic approximation

$$
M\left(\tilde{z}_{\varepsilon}(t)\right)=M\left(z^{*}\right)+\varepsilon M^{\prime}\left(z^{*}\right) z_{1}(t)+\frac{\varepsilon^{2}}{2} M^{\prime}\left(z^{*}\right) z_{2}(t)+\frac{\varepsilon^{2}}{2} M^{\prime \prime}\left(z^{*}\right)\left(z_{1}(t)\right)^{2}+\delta(\varepsilon ; t) .
$$

Here $\delta(\varepsilon, t)$ is continuous in $t \in[0, T], T$-periodic, and $\delta(\varepsilon ; t) / \varepsilon^{2} \rightarrow 0$ uniformly when $\varepsilon \rightarrow 0$. In this way we come up with the equation

$$
\begin{aligned}
\mathcal{D} n^{*} & (a)+\varepsilon \mathcal{D} n_{1}(t, a)+\frac{\varepsilon^{2}}{2} \mathcal{D} n_{2}(t, a) \\
= & h_{n}(a) n^{*}(a) \\
& +\varepsilon\left[h_{n}(a) n_{1}(t, a)+h_{z}(a) z_{1}(t)+h_{v}(a) w(t)\right] \\
& \left.+\frac{\varepsilon^{2}}{2}\left[h_{n}(a) n_{2}(t, a)+h_{z}(a) z_{2}(t)+2\left(h_{z n} z_{1}(t)+h_{v n}(a) w(t)\right) n_{1}(t, a)+h_{z z}\left(z_{1}(t)\right)^{2}\right)\right]+r(\varepsilon ; t, a),
\end{aligned}
$$

where

$$
\begin{gathered}
h_{n}(a):=-\left(\mu(a)+M\left(z^{*}\right)+v^{*} \chi(a)\right), \quad h_{z}(a):=-M^{\prime}\left(z^{*}\right) n^{*}(a), \quad h_{v}(a):=-\chi(a) n^{*}(a), \\
h_{z n}:=-M^{\prime}\left(z^{*}\right), \quad h_{z z}(a)=-M^{\prime \prime}\left(z^{*}\right) n^{*}(a), \quad h_{v n}(a)=-\chi(a),
\end{gathered}
$$

and the residual $r(\varepsilon ; t, a)$ is measurable, bounded, $T$-periodic, and $r(\varepsilon ; t, a) / \varepsilon^{2} \rightarrow 0$ uniformly when $\varepsilon \rightarrow 0$.

Separately equalizing to zero the first and second order terms (the zero-order term is zero due to equations (3.1)-(3.3) that $\left(n^{*}, z^{*}\right)$ satisfies) we come up with the following two systems:

$$
\begin{aligned}
\mathcal{D} n_{1}(t, a) & =h_{n}(a) n_{1}(t, a)+h_{z}(a) z_{1}(t)+h_{v}(a) w(t), \\
n_{1}(0, a) & =n_{1}(T, a), \\
n_{1}(t, 0) & =\int_{0}^{A} \beta(a) n_{1}(t, a) \mathrm{d} a, \\
z_{1}(t) & =\int_{0}^{A} \gamma(a) n_{1}(t, a) \mathrm{d} a,
\end{aligned}
$$


and

$$
\begin{aligned}
\mathcal{D} n_{2}(t, a) & =h_{n}(a) n_{2}(t, a)+h_{z}(a) z_{2}(t)+2\left(h_{z n} z_{1}(t)+h_{v n}(a) w(t)\right) n_{1}(t, a)+h_{z z}(a) z_{1}(t)^{2}, \\
n_{2}(0, a) & =n_{2}(T, a), \\
n_{2}(t, 0) & =\int_{0}^{A} \beta(a) n_{2}(t, a) \mathrm{d} a, \\
z_{2}(t) & =\int_{0}^{A} \gamma(a) n_{2}(t, a) \mathrm{d} a .
\end{aligned}
$$

Proposition 4.2. System (4.6)-(4.9) has a solution $\left(n_{1}, z_{1}\right)$ in the form

$$
\begin{aligned}
n_{1}(t, a) & =n_{c}(a) \cos (\Omega t)+n_{s}(a) \sin (\Omega t), \\
z_{1}(t) & =z_{c} \cos (\Omega t)+z_{s} \sin (\Omega t),
\end{aligned}
$$

where $\left(n_{c}, n_{s}, z_{c}, z_{s}\right)$ is the unique solution of the following functional-ODE:

$$
\begin{gathered}
\frac{\mathrm{d} n_{c}(a)}{\mathrm{d} a}+\Omega n_{s}(a)=h_{n}(a) n_{c}(a)+h_{z}(a) z_{c}, \\
\frac{\mathrm{d} n_{s}(a)}{\mathrm{d} a}-\Omega n_{c}(a)=h_{n}(a) n_{s}(a)+h_{z}(a) z_{s}+h_{v}(a) \\
z_{c}=\int_{0}^{A} \gamma(a) n_{c}(a) \mathrm{d} a, \quad z_{s}=\int_{0}^{A} \gamma(a) n_{s}(a) \mathrm{d} a, \\
n_{c}(0)=\int_{0}^{A} \beta(a) n_{c}(a) \mathrm{d} a, \quad n_{s}(0)=\int_{0}^{A} \beta(a) n_{s}(a) \mathrm{d} a .
\end{gathered}
$$

Moreover, system (4.10)-(4.13) has a solution $n_{2}$ in the form

$$
n_{2}(t, a)=n_{0}(a)+\sum_{k=1}^{2}\left(n_{c k}(a) \cos (k \Omega t)+n_{s k}(a) \sin (k \Omega t)\right),
$$

where $n_{0}, n_{c k}, n_{s k}$ are appropriate absolutely continuous functions and $n_{0}$ is the unique solution of the following functional-ODE:

$$
\begin{aligned}
\frac{\mathrm{d} n_{0}(a)}{\mathrm{d} a} & =h_{n}(a) n_{0}(a)+h_{z}(a) z_{0}+h_{0}(a), \\
z_{0} & =\int_{0}^{A} \gamma(a) n_{0}(a) \mathrm{d} a, \\
n_{0}(0) & =\int_{0}^{A} \beta(a) n_{0}(a) \mathrm{d} a,
\end{aligned}
$$

where $h_{0}(a):=h_{z n}\left(z_{c} n_{c}(a)+z_{s} n_{s}(a)\right)+h_{v n}(a) n_{s}(a)+h_{z z}(a) \frac{z_{c}^{2}+z_{s}^{2}}{2}$.

The proof of this proposition is given in the Appendix.

Below we fix the solutions $n_{1}$ and $n_{2}$ specified in the above proposition (in fact, they are the unique solutions of the respective systems, but this fact is not obvious and we do not need it).

Now we compare the functions $n^{*}$ and $\bar{n}_{\varepsilon}$, reminding that $n^{*}(a)>\nu$ for some $\nu>0$ (thus it is the unique strictly positive solution of (2.6)-(2.9) for control $\left.v(t)=v^{*}\right)$, and $\bar{n}_{\varepsilon}$ is the maximal non-negative solution of the same system with control $v(t)=v^{*}+\varepsilon w(t)$. Observe, that $n^{*}$ solves the equation

$$
\mathcal{D} \bar{n}(t, a)=-\left(\mu(a)+M(z(t))+v^{*} \chi(a)\right) \bar{n}(t, a)-\varepsilon \chi(a) w(t) \bar{n}(t, a), \quad(t, a) \in[0, T] \times[0, A]
$$


(together with $(2.6)-(2.9))$ with $\varepsilon=0$. For the last term we have $|\varepsilon \chi(a) w(t)| \leq \varepsilon\|\chi\|_{\infty}$. Then, thanks to Assumption $(\mathrm{H})$, we can apply Theorem 5F.4 in [8] (implicit function theorem with strong metric regularity). In our case it implies that there exist $\varepsilon_{1}>0$ and $\delta_{1}>0$ such that for every $\varepsilon \in\left(0, \varepsilon_{1}\right]$ system $(4.23),(2.6)-(2.9))$ has a unique solution $n_{\varepsilon}^{*}(t, a)$ in the $\delta_{1}$-neighborhood of $v^{*}$ (in $\left.L_{\infty}\right)$ and

$$
\left\|n^{*}-n_{\varepsilon}^{*}\right\|_{\infty} \leq \varepsilon d\left\|_{\chi}\right\|_{\infty}:=\varepsilon c_{1},
$$

where $d$ is the constant from Assumption $(\mathrm{H})$. In particular, $n_{\varepsilon}^{*}(t, a)>0$ for all sufficiently small $\varepsilon$ (namely such that $\left.\varepsilon d\|\chi\|_{\infty}<\nu\right)$. However, $\bar{n}_{\varepsilon}$ is the maximal non-negative solution of the same system, and the latter has at most one non-negative solution which is not identically zero. Hence $\bar{n}_{\varepsilon}=n_{e}^{*}$ and

$$
\left\|n^{*}-\bar{n}_{\varepsilon}\right\|_{\infty} \leq \varepsilon c_{1} .
$$

Now we consider system (4.23), (2.6)-(2.9)) (satisfied by $\bar{n}_{\varepsilon}$ ) and the same system with a disturbance $m(t, a)=r(\varepsilon ; t, a)$ (which is satisfied by $\tilde{n}_{\varepsilon}$ ). Applying again [8, Theorem 5F.4] (we skip the details, which are routine, but require some additional work), we obtain that for all sufficiently small $\varepsilon>0$

$$
\left\|\bar{n}_{\varepsilon}-\tilde{n}_{\varepsilon}\right\|_{\infty} \leq \varepsilon c_{2},
$$

where $c_{2}$ is independent of $\varepsilon$. Thus, we justify the representation

$$
\bar{n}_{\varepsilon}(t, a)=n^{*}(a)+\varepsilon n_{1}(t, a)+\frac{\varepsilon^{2}}{2} n_{2}(t, a)+o\left(\varepsilon^{2}\right) .
$$

Here and below we denote by $o(\varepsilon)$ any bounded and measurable function of $(t, a) \in[0, T] \times[0, A]$ such that $o(\varepsilon) / \varepsilon \rightarrow 0$ with $\varepsilon \rightarrow 0$, uniformly in $(t, a) \in[0, T] \times[0, A]$.

Now we are prepared to investigate the dependence of $\hat{J}(\varepsilon)$ on $\varepsilon$. Let us abbreviate $\varphi(a):=\gamma(a) \chi(a)$. From (4.2) we have

$$
\begin{aligned}
\hat{J}(\varepsilon)= & \frac{1}{T} \int_{0}^{T}\left(\int_{0}^{A} \varphi(a)\left(n^{*}(a)+\varepsilon n_{1}(t, a)+\frac{\varepsilon^{2}}{2} n_{2}(t, a)+o\left(\varepsilon^{2}\right)\right) \mathrm{d} a-c\right)\left(v^{*}+\varepsilon w(t)\right) \mathrm{d} t \\
= & \left(\int_{0}^{A} \varphi(a) n^{*}(a) \mathrm{d} a-c\right) v^{*}+\frac{\varepsilon}{T}\left(\int_{0}^{A} \varphi(a) n^{*}(a) \mathrm{d} a-c\right) \int_{0}^{T} w(t) \mathrm{d} t \\
& +\frac{\varepsilon}{T} \int_{0}^{A} \varphi(a) \int_{0}^{T} n_{1}(t, a) \mathrm{d} t \mathrm{~d} a v^{*} \\
& +\frac{\varepsilon^{2}}{T} \int_{0}^{A} \varphi(a) \int_{0}^{T} n_{1}(t, a) w(t) \mathrm{d} t \mathrm{~d} a+\frac{\varepsilon^{2}}{2 T} \int_{0}^{A} \varphi(a) \int_{0}^{T} n_{2}(t, a) \mathrm{d} t \mathrm{~d} a v^{*}+o\left(\varepsilon^{2}\right) .
\end{aligned}
$$

We observe that the first term in the last right-hand side is just $J\left(T, v^{*}\right)$, the second integral in the second term is zero due to the specific form of $w$ in (4.1), the inner integral in the third term is also zero due to the representation (4.14) of $n_{1}$. Moreover, according to (4.19), $\int_{0}^{T} n_{2}(t, a) \mathrm{d} t=\int_{0}^{T} n_{0}(a) \mathrm{d} t$. Thus we obtain that

$$
\hat{J}(\varepsilon)=J\left(T, v^{*}\right)+\frac{\varepsilon^{2}}{T} \int_{0}^{A} \varphi(a) \int_{0}^{T} n_{1}(t, a) w(t) \mathrm{d} t \mathrm{~d} a+\frac{\varepsilon^{2}}{2} \int_{0}^{A} \varphi(a) n_{0}(a) \mathrm{d} a v^{*}+o\left(\varepsilon^{2}\right) .
$$

Now, we again take into account the specific form of $w$, the representation (4.14) of $n_{1}$, and the fact that $\int_{0}^{T} \sin (\Omega t) \cos (\Omega t) \mathrm{d} t=0$ and $\int_{0}^{T} \sin ^{2}(\Omega t) \mathrm{d} t=T / 2$, which yields

$$
\hat{J}(\varepsilon)=J\left(T, v^{*}\right)+\frac{\varepsilon^{2}}{2} \int_{0}^{A} \gamma(a) \chi(a)\left(n_{s}(a)+v^{*} n_{0}(a)\right) \mathrm{d} a+o\left(\varepsilon^{2}\right) .
$$


Thus we obtain the following properness test (in the terminology of [7]): if

$$
\hat{J}^{\prime \prime}:=\int_{0}^{A} \gamma(a) \chi(a)\left(n_{s}(a)+v^{*} n_{0}(a)\right) \mathrm{d} a>0
$$

then there is a proper periodic control (namely, $v^{*}+\varepsilon w(t)$ for all sufficiently small $\varepsilon$ ) which is better than the optimal constant one. Here $n_{s}$ is uniquely determined by the functional-ODE system (4.15)-(4.18), and $n_{0}$ is the unique solution of (4.20)-(4.22).

Remark 4.3. From a numerical point of view, a straightforward alternative to the properness test developed above will be to compare $J\left(T, v^{*}\right)$ and $J\left(T, v^{*}+\varepsilon w(\cdot)\right)$ by direct numerical calculations. However, this will require solving the distributed system $(2.6)-(2.9)$. Since, as seen above, $J\left(T, v^{*}+\varepsilon w(\cdot)\right)-J\left(T, v^{*}\right)$ is of order $\varepsilon^{2}$, the accuracy of the solution has to be significantly higher, which may be problematic, since it is not known in advance how small $\varepsilon$ should be taken. The advantage of the properness test is that its check involves only solving ODEs, and the accuracy should be high enough only to determine the sign of $\hat{J}^{\prime \prime}$, which may be well positive. The parameter $\varepsilon$ is not involved in the calculations, at all.

In the next subsection we perform the properness test for several test examples and identify cases where a periodic control strictly dominates any constant one (due to (4.24)).

\subsection{Numerical properness test}

In the numerical tests below we take as benchmark data mortality rate $\mu(a)=0.005$, mortality rate due to competition $M(z)=0.001 z$, and fertility and size functions $\beta(a)$ and $\gamma(a)$ as in Fig. 4.2.

We consider three different scenarios for the mode of selective fishing, $\chi(a)$. They are represented on the left plots in Fig. 3. In all cases we first find numerically the optimal constant harvesting effort $v^{*}$ as described in Section 3, then we perform the properness test (4.24) (by solving systems (4.15)-(4.18) and (4.20)-(4.22)) for various values of $\Omega=\frac{2 \pi}{T}$ with $T \in(0, \bar{T}]$, where $\bar{T}=10$.

From the right plots in Fig. 3 we observe the following:

(i) When fishing is not selective $(\chi(a) \equiv 1$ - see Fig. 3 (a) $)$ then the properness test fails: $\hat{J}^{\prime \prime} \leq 0$ for all periods $T \leq \bar{T}$. In the next section we prove that constant fishing is really optimal in this case.

(ii) If the fishing is selective and applies to fish in fertile ages $(a \in[10,20]$ in the benchmark), but not before maturation (that is, for $a<10$ in the benchmark) then the properness test clearly shows that a sinusoidal periodic fishing rate, for example, with period $T=6.3$ is better than the optimal constant one (see Fig. $3(\mathrm{~b})$ ). Observe, that $\hat{J}^{\prime \prime} \approx 2.9$ in this case, while the accuracy of its evaluation is a decimal fraction.

(iii) If fishing is applied only at old non-fertile ages ( $a>20$ in our benchmark), then the properness test fails again $\left(\hat{J}^{\prime \prime}<0\right.$ for all $\left.T \leq \bar{T}\right)$, see Fig. 3 (c). In fact, in this case constant fishing with maximal effort $V$ may be optimal if the cost of fishing is disregarded (see the right plot in Fig. 1 for $c=0$ ).

\section{The case of non-selective fishing}

In this section we consider the case of non-selective fishing: $\chi(a) \equiv \chi \in(0, \infty)$. In order to investigate this case analytically, we assume that only measurable $T$-periodic controls $v(a) \in[0, V]$ are admissible, where $T \leq \bar{T}$ (see Assumption (A1)). The goal of the section is to prove that in the case of non-selectivity the fishing problem considered in this paper has a constant solution.

Instead of Theorem 2.1, which requires continuity of the control function $v(\cdot)$, we may apply Theorem 2.1 in [3] (see Remark 2.2 about the assumptions). It applies for a measurable, bounded and $T$-periodic control function $v(\cdot), T \in(0, \bar{T}]$, and claims the same as Theorem 2.1, provided that $\chi$ is constant. 

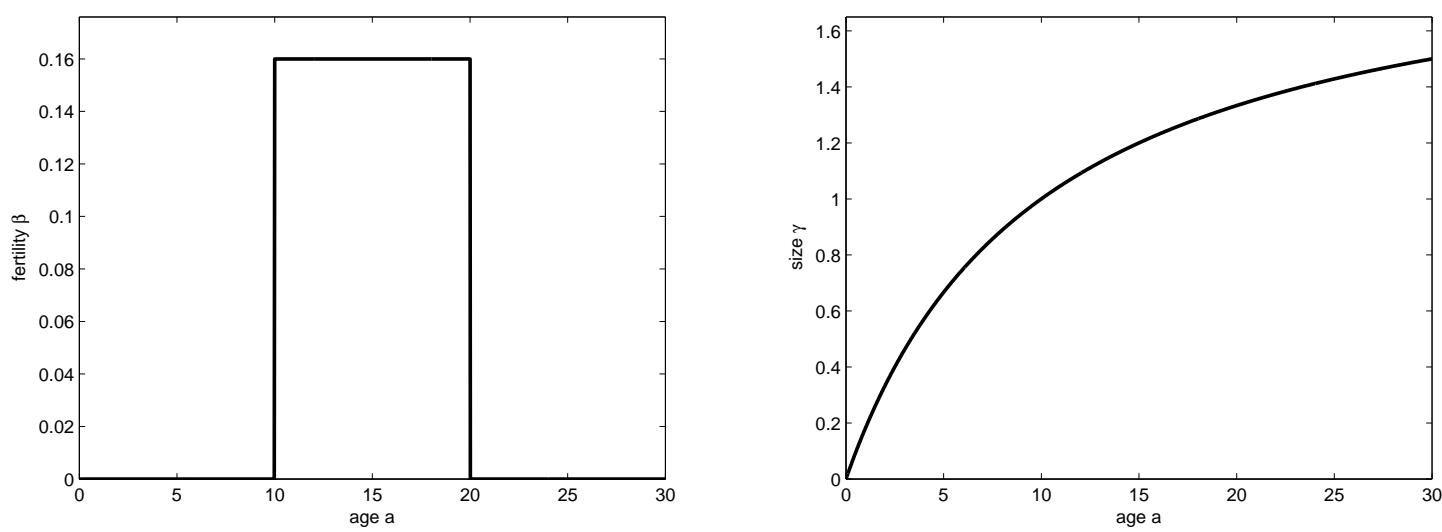

Figure 2. Fertility $\beta(a)$ (left) and size $\gamma(a)=\frac{2 a}{10+a}$ (right) depending on the age $a$.

Again, system (2.6)-(2.9) has at most two non-negative solutions, one of which is identically zero. If $\bar{n}$ is the maximal non-negative solution, then our objective functional (2.5) in the class of all $T$-periodic controls takes again the form (4.2), with the constant $\chi$ and any measurable $v:[0, T] \rightarrow[0, V], T \in(0, \bar{T}]$.

Following [4] (see also [12,14]) and making use of the stationarity of the vital rates in our model, we represent $\bar{n}(t, a)$ in the form

$$
\bar{n}(t, a)=y(t) n(a),
$$

where both $y$ and $n$ are absolutely continuous and $n(0)=1$. To do this we insert the above expression for $\bar{n}$ in (2.6)-(2.9) and obtain that $y$ and $n$ should satisfy the system

$$
\begin{aligned}
y^{\prime}(a) n(a)+y(a) n^{\prime}(a) & =-(\mu(a)+M(\bar{z}(t)+\chi v(t)+\alpha-\alpha) y(a) n(a), \\
y(0) n(a) & =y(T) n(a), \\
y(t) n(0) & =y(t) \int_{0}^{A} \beta(a) n(a) \mathrm{d} a, \\
z(t) & =y(t) \int_{0}^{A} \gamma(a) n(a) \mathrm{d} a,
\end{aligned}
$$

where $\alpha$ is a real number, viewed as a parameter to be chosen. Formally separating the age-dependent and the time-dependent terms we obtain the following two systems:

$$
\begin{aligned}
n^{\prime}(a) & =-(\mu(a)+\alpha) n(a), \\
1 & =\int_{0}^{A} \beta(a) n(a) \mathrm{d} a \quad(=n(0)),
\end{aligned}
$$

and

$$
\begin{aligned}
& y^{\prime}(t)=-(M(z y(t))+\chi v(t)-\alpha) y(t), \\
& y(0)=y(T)
\end{aligned}
$$

where $z$ is defined as

$$
z=\int_{0}^{A} \gamma(a) n(a) \mathrm{d} a .
$$

Now we chose the parameter $\alpha$ so that system (5.2), (5.3) has a solution. If $n$ is a solution with $n(0)=1$, then we have

$$
n(a)=e^{-\int_{0}^{a} \mu(\eta) \mathrm{d} \eta-\alpha a},
$$



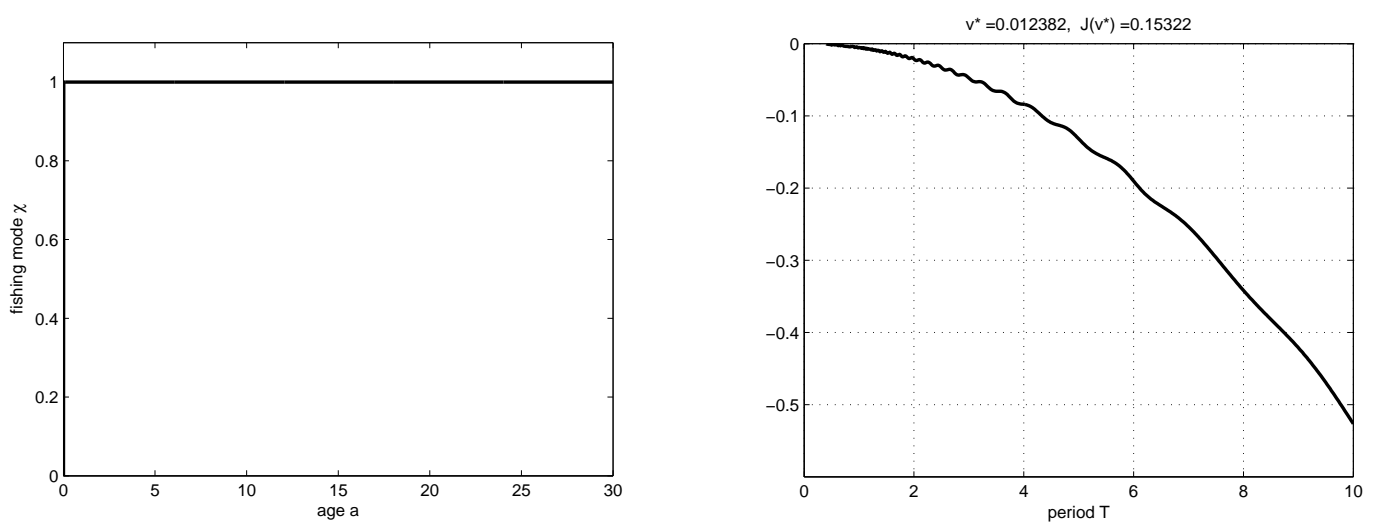

a)

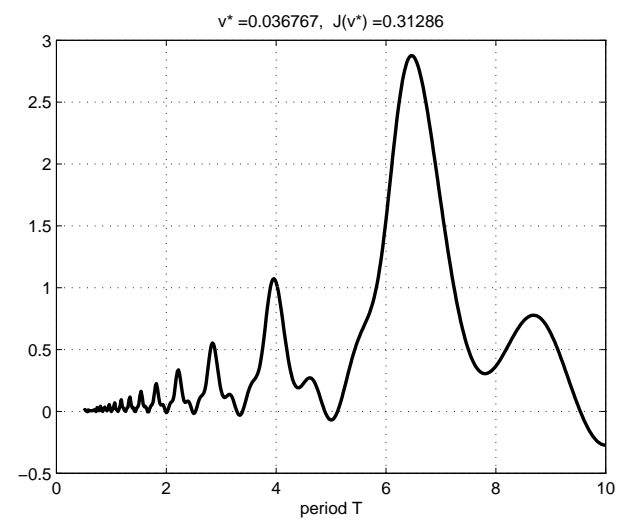

b)
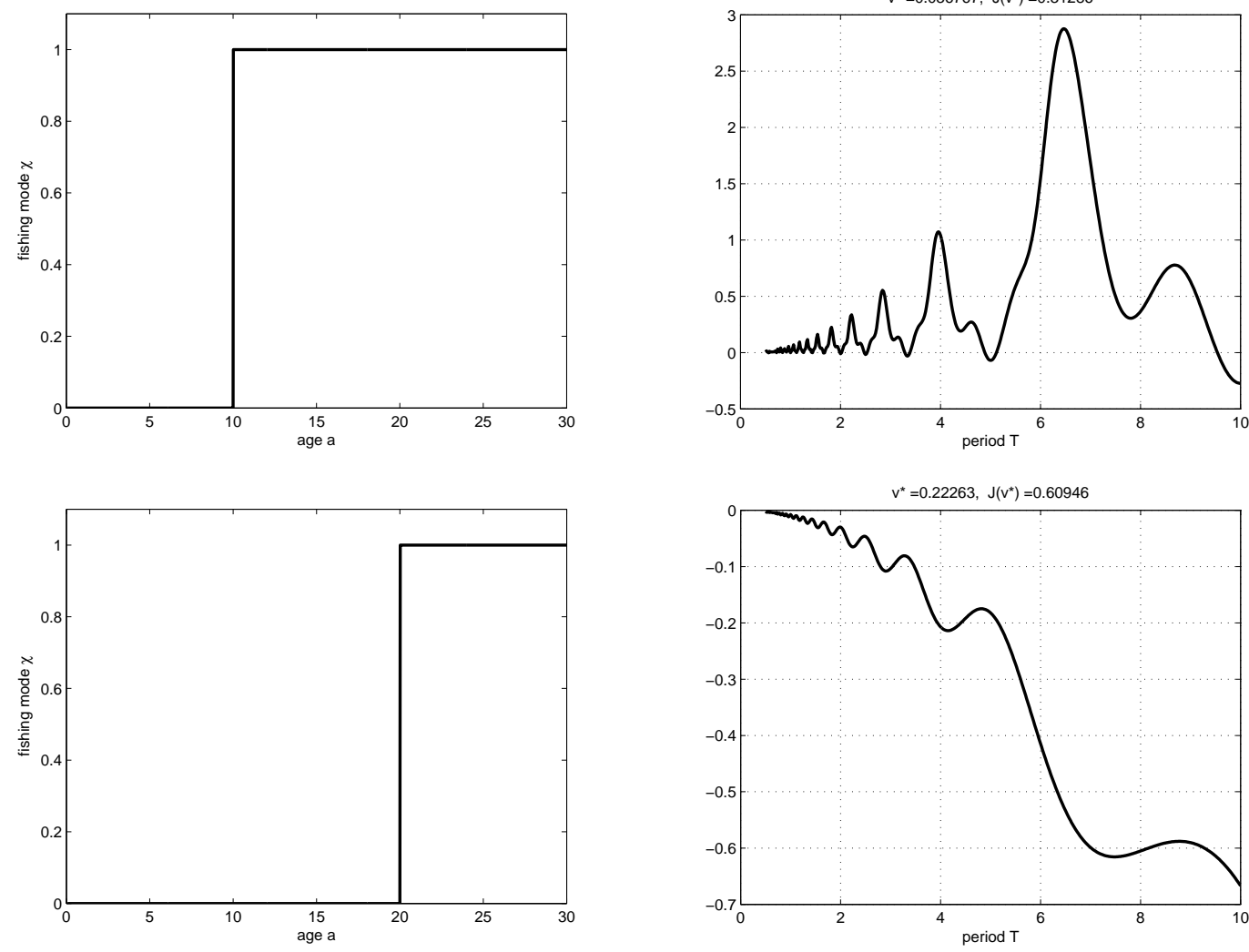

Figure 3. Fishing mode depending on the age $a$ (left) and the second derivative of objective functional, $J^{\prime \prime}$, w.r. to $\varepsilon$ at $\varepsilon=0$ (right) depending on the period $T$.

and to ensure (5.3) it is necessary and sufficient that

$$
\int_{0}^{A} \beta(a) e^{-\int_{0}^{a} \mu(\eta) \mathrm{d} \eta-\alpha a} \mathrm{~d} a=1
$$

Due to Assumption (A2), for $\alpha=0$ the left-hand side is strictly bigger than 1. Since the left-hand side is strictly decreasing and continuous in $\alpha$, and converges to zero when $\alpha \rightarrow+\infty$, the above equation has a unique solution which will be denoted further in this section just by $\alpha$. Clearly, $\alpha>0$. For the 
chosen $\alpha$ system (5.2), (5.3) has the unique solution (5.7). Observe that $\alpha$ does not depend on the chosen normalization $n(0)=1$.

Now we consider system (5.4), (5.5), where $z$ is defined by (5.6). Clearly, $z>0$, since $n(a)>0$ for all a. According to [3, Lemma 2.2] we have the following alternative:

(i) If $\chi \int_{0}^{T} v(t) \mathrm{d} t<\alpha T$, then system (5.4), (5.5) has a unique non-trivial non-negative solution. In this case $\bar{n}(t, a)=y(t) n(a)$ is the maximal non-negative solution of (2.6)-(2.9).

(ii) If $\chi \int_{0}^{T} v(t) \mathrm{d} t \geq \alpha T$, then $y(t)=0$ is the unique nonnegative solution of system (5.4), (5.5). In this case $\bar{n}=0$ is the maximal non-negative solution of (2.6)-(2.9).

Accordingly, we obtain the following representation of the objective function $(4.2)$ for $T \in(0, \bar{T}]$ and a measurable control $v$ :

$$
J(T, v)=\frac{1}{T} \int_{0}^{T}(\chi z y(t)-c) v(t) \mathrm{d} t
$$

where $y$ is the maximal solution of $(5.4),(5.5)$. This is to be maximized under the constraints $T \in(0, \bar{T}]$, $v(t) \in[0, V]$.

Remark 5.1. Since the half-lines $y \leq 0$ and $y \geq 0$ are invariant with respect to (5.4), any solution $y$ is either non-negative or non-positive. Since $z>0$, a non-trivial non-positive solution cannot be optimal. Thus the following problem is equivalent to our original one: maximize (5.8) under the constraints $T \in(0, \bar{T}], v(t) \in[0, V]$, where $y$ is some solution of $(5.4),(5.5)$. This reformulation is essential for the utilization of the paper [11] below.

Since $J(T, 0)=0$, the supremum of $J(T, v)$ over the admissible $(T, v)$, denoted by $J^{*}$, is non-negative. If $J^{*}=0$, then $v=0$ is optimal. Then we are done - an optimal constant harvesting exists.

Therefore, further we assume that $J^{*}>0$. Take a maximizing sequence $\left(T_{k}, v_{k}\right)$, where we may assume that $T_{k}$ converges. Let us consider the following two cases.

Case 5.2. $T_{k} \rightarrow T_{0}>0$. Then we can extend or restrict each $v_{k}$ to $\left[0, T_{0}\right]$ and (thanks to the BanachAlaoglu theorem) extract a subsequence, denoted again by $v_{k}$, which converges to some $v_{0} \in L_{\infty}\left(0, T_{0}\right)$ in the weak-* topology in $L_{\infty}=\left(L_{1}\right)^{*}$. Since equation (5.4) is linear in $v$, by a standard argument $y_{k} \rightarrow y_{0}$ uniformly, where $y_{k}$ is the maximal solution of (5.4), (5.5) corresponding to $v_{k}$, and $y_{0}$ is a solution corresponds to $v_{0}$.

Due to $y_{k}(t)>0$ we have $y(t) \geq 0$. If $y_{0}$ is not the maximal solution, then $y=0$, which means that $y_{k} \rightarrow 0$ uniformly. This implies $J\left(T_{k}, v_{k}\right) \rightarrow 0$, which contradicts $J^{*}>0$. Thus $y_{0}$ is the maximal solution of (5.4), (5.5) corresponding to $v_{0}$. Hence,

$$
J\left(T_{0}, v_{0}\right)=\frac{1}{T_{0}} \int_{0}^{T_{0}}\left(\chi z y_{0}(t)-c\right) v_{0}(t) \mathrm{d} t .
$$

Passing to the limit in (5.8) with $\left(T_{k}, v_{k}, y_{k}\right)$, which is possible due to the linearity of the integrant w.r.t. $v(t)$, we obtain the right-hand side of the last equality, hence $J^{*}=J\left(T_{0}, v_{0}\right)$. We obtained an optimal solution $\left(T_{0}, v_{0}\right)$.

If $v_{0}$ is constant, then we are done. Assume that it is not. Then we apply to problem (5.8), (5.4), (5.5) Theorem 2.1 in [11] (see Remark 5.1). It claims that there exists an optimal solution $\left(T_{0}^{1}, v_{0}^{1}\right)$ with $T_{0}^{1} \leq T_{0} / 2$. If $v_{0}^{1}$ is not constant (otherwise we are done), then we may continue applying Theorem 2.1 in [11] until we obtain a constant optimal control, or to infinity. In the latter case we obtain a sequence of optimal controls $\left(T_{0}^{k}, v_{0}^{k}\right)$ such that $T_{0}^{k} \rightarrow 0$.

Case 5.3. $T_{k} \rightarrow 0$. See further. 
In both cases 5.2 and 5.3 we have a maximizing sequence $\left(T_{k}, v_{k}\right)$ with $T_{k} \rightarrow 0$. Moreover, the corresponding maximal $y_{k}$ are strictly positive due to $J^{*}>0$. We construct an optimal constant fishing on every interval $[0, T]$ with $T \in(0, \bar{T}]$. To do this we fix such a $T$, define $N_{k}:=\left[T / T_{k}\right]$ (the integer part of $\left.\left[T / T_{k}\right]\right)$ and the control

$$
\left.\hat{v}_{k}(t)=v_{k}\left(t-i T_{k}\right) \quad \text { for } t \in\left[i T_{k},(i+1) T_{k}\right)\right], \quad i=0, \ldots, N_{k}-1 .
$$

Due to the $T_{k}$-periodicity of $y_{k}$, the function

$$
\left.\hat{y}_{k}(t)=y_{k}\left(t-i T_{k}\right) \quad \text { for } t \in\left[i T_{k},(i+1) T_{k}\right)\right], \quad i=0, \ldots, N_{k}-1 .
$$

solves (5.4), (5.5), and it is its maximal solution since it is strictly positive (as $y_{k}$ are). Then it is straightforward to show that with $\hat{T}_{k}=N_{k} T_{k}$ we have that

$$
\left.J\left(\hat{T}_{k}, \hat{v}_{k}\right)\right)=J\left(T_{k}, v_{k}\right) \rightarrow J^{*} .
$$

That is, $\left(\hat{T}_{k}, \hat{v}_{k}\right)$ is another maximizing sequence with $\hat{T}_{k} \rightarrow T>0$. We may repeat the first part of the analysis in Case 5.2, where we show that the weak-* limit (of a subsequence) of $v_{k}$ is a solution, together with $T$. This time, however, any weak-* limit is constant (and equals some condensation point of $\left.\frac{1}{T_{k}} \int_{0}^{T_{k}} v_{k}(t) \mathrm{d} t\right)$, due to the definition of $\hat{v}_{k}$.

Thus we have shown that on our basic assumptions (A1)-(A4) a constant optimal harvesting exists if the fishing is not selective.

\section{Concluding remarks}

The main result in this paper shows that if the age-structure of the fish is taken into account in a long-run optimization of the averaged profit, then a periodic fishing effort can be strictly better than any constant one. For this effect to occur, it is essential that the fishing is selective: only fish of prescribed ages/sizes is harvested. This selectivity may be imposed by regulation or by market requirements, therefore it is endogenous for the considered model. On the other hand, discrete-time models are investigated in the literature, where the minimal age of fishing is regarded as another decision variable (knife-edge selectivity, see. e.g [13] and the references there), along with the fishing effort. Although the present paper does not present results for such models, our numerical results (based on the developed properness test) show that a proper periodic fishing can be strictly dominating any constant one also if the lower age of fishing is chosen optimally.

\section{Appendix}

Below we present the proof of Proposition 4.2.

Part 1. First we shall prove that system (4.15)-(4.18) has a unique solution. The fundamental matrix solution of the homogeneous part of (4.15), (4.16) is

$$
e^{\int_{0}^{a} h_{n}(\eta) \mathrm{d} \eta} S(a), \quad \text { where } \quad S(a):=\left(\begin{array}{cc}
\cos (\Omega a) & -\sin (\Omega a) \\
\sin (\Omega a) & \cos (\Omega a)
\end{array}\right) .
$$

Then the solution of (4.15), (4.16) takes the form

$$
\begin{aligned}
& \left(\begin{array}{c}
n_{c}(a) \\
n_{s}(a)
\end{array}\right)=e^{\int_{0}^{a} h_{n}(\eta) \mathrm{d} \eta} S(a)\left(\left(\begin{array}{c}
n_{c}(0) \\
n_{s}(0)
\end{array}\right)+\int_{0}^{a} \frac{S(\eta)^{-1}}{e^{\int_{0}^{a} h_{n}(\eta) \mathrm{d} \eta}}\left(h_{z}(\eta)\left(\begin{array}{c}
z_{c} \\
z_{s}
\end{array}\right)+h_{v}(\eta)\left(\begin{array}{l}
0 \\
1
\end{array}\right)\right) \mathrm{d} \eta\right) \\
& =n^{*}(a) S(a)\left(\frac{1}{n^{*}(0)}\left(\begin{array}{c}
n_{c}(0) \\
n_{s}(0)
\end{array}\right)-M^{\prime}\left(z^{*}\right) \int_{0}^{a} S(\eta)^{T} \mathrm{~d} \eta\left(\begin{array}{c}
z_{c} \\
z_{s}
\end{array}\right)-\int_{0}^{a} S(\eta)^{T} \chi(\eta) \mathrm{d} \eta\left(\begin{array}{l}
0 \\
1
\end{array}\right)\right)
\end{aligned}
$$


where the superscript ${ }^{T}$ denotes transposition, and we use that $e^{\int_{0}^{a} h_{n}(\eta) \mathrm{d} \eta} n^{*}(0)=n^{*}(a)$.

The integral of $S$ can be expressed as follows

$$
\int_{0}^{a} S(\eta)^{T} \mathrm{~d} \eta=\frac{1}{\Omega}\left(\begin{array}{cc}
\sin (\Omega a) & -\cos (\Omega a) \\
\cos (\Omega a) & \sin (\Omega a)
\end{array}\right)-\frac{1}{\Omega}\left(\begin{array}{cc}
0 & -1 \\
1 & 0
\end{array}\right),
$$

so that

$$
S(a) \int_{0}^{a} S(\eta)^{T} \mathrm{~d} \eta=\frac{1}{\Omega}\left(\begin{array}{cc}
0 & -1 \\
1 & 0
\end{array}\right)\left(\left(\begin{array}{ll}
1 & 0 \\
0 & 1
\end{array}\right)-S(a)\right) .
$$

Notice that the rotation matrix $S$ belongs to the matrix space generated by linear combinations of $i d e n t i t y$ matrix I and unit symplectic matrix E:

$$
I=\left(\begin{array}{ll}
1 & 0 \\
0 & 1
\end{array}\right), \quad E=\left(\begin{array}{cc}
0 & -1 \\
1 & 0
\end{array}\right)
$$

e.g. $S(a)=\cos (\Omega a) I+\sin (\Omega a) E$. It is easy to see that the product of matrices from this space is commutative and these matrices have non-negative determinant that becomes zero only when all matrix elements are zero. Now, we can express the following integrals, where the matrices $S_{\beta}$ and $S_{\gamma}$ belong to the aforementioned space:

$$
\begin{array}{r}
\int_{0}^{A} \beta(a) n^{*}(a) S(a) \int_{0}^{a} S(\eta)^{T} \mathrm{~d} \eta \mathrm{d} a=\frac{n^{*}(0)}{\Omega} E\left(I-S_{\beta}\right), \quad S_{\beta}:=\frac{1}{n^{*}(0)} \int_{0}^{A} \beta(a) n^{*}(a) S(a) \mathrm{d} a, \\
\int_{0}^{A} \gamma(a) n^{*}(a) S(a) \int_{0}^{a} S(\eta)^{T} \mathrm{~d} \eta \mathrm{d} a=\frac{n^{*}(0)}{\Omega} E\left(\frac{z^{*}}{n^{*}(0)} I-S_{\gamma}\right) \quad S_{\gamma}:=\frac{1}{n^{*}(0)} \int_{0}^{A} \gamma(a) n^{*}(a) S(a) \mathrm{d} a .
\end{array}
$$

Using these expressions we can write conditions (4.17)-(4.18) in the form of the following linear system

$$
\left(\begin{array}{cc}
I-S_{\beta} & m E\left(I-S_{\beta}\right) \\
-S_{\gamma} & I+m E\left(\frac{z^{*}}{n^{*}(0)} I-S_{\gamma}\right)
\end{array}\right)\left(\begin{array}{c}
n_{c}(0) \\
n_{s}(0) \\
z_{c} \\
z_{s}
\end{array}\right)=\left(\begin{array}{c}
-\int_{0}^{A} \beta(a) n^{*}(a) S(a) \int_{0}^{a} S(\eta)^{T} \chi(\eta) \mathrm{d} \eta \mathrm{d} a \\
-\int_{0}^{A} \gamma(a) n^{*}(a) S(a) \int_{0}^{a} S(\eta)^{T} \chi(\eta) \mathrm{d} \eta \mathrm{d} a
\end{array}\right)\left(\begin{array}{l}
0 \\
1
\end{array}\right),
$$

where $m=\frac{n^{*}(0) M^{\prime}\left(z^{*}\right)}{\Omega}$. The unique solution exists if and only if the matrix of system (6.4) is invertible. Due to condition (3.6), which reads in the current notations as

$$
\frac{1}{n^{*}(0)} \int_{0}^{A} \beta(a) n^{*}(a) \mathrm{d} a=1,
$$

and the definition of $S_{\beta}$, the absolute values of all its elements are less than 1 . Thus, all diagonal elements of $I-S_{\beta}$ are positive. Recalling that such matrix is degenerate only if all its elements are zero, we have $\operatorname{det}\left(I-S_{\beta}\right)>0$. If we multiply system (6.4) from the left by the non-degenerate matrix:

$$
\left(\begin{array}{cc}
I & 0 \\
S_{\gamma}\left(I-S_{\beta}\right)^{-1} & I
\end{array}\right)
$$

then from the commutativity of the blocks, we obtain the following matrix:

$$
\left(\begin{array}{cc}
I-S_{\beta} & m E\left(I-S_{\beta}\right) \\
0 & I+\frac{z^{*} M^{\prime}\left(z^{*}\right)}{\Omega} E
\end{array}\right)
$$

The determinant of the resulting upper triangular block-matrix is equal to the product of the determinants of its diagonal blocks, which are strictly positive. Hence, the matrix of system (6.4) is invertible and there exists a unique solution of system (4.15)-(4.18). 
The obtained $n_{1}$ and $z_{1}$ solve (4.6)-(4.9), as a direct substitution shows.

The procedure for obtaining a solution of (4.10)-(4.13) in the form (4.19) is similar. The equations for the coefficients $n_{0}, n_{c k}$ and $n_{s k}$ in (4.19) can be obtained by formal substitution in (4.10)-(4.13). The resulting system (out of which only $n_{0}$ is needed for our further analysis) is 4-dimensional, but its unique solvability can be proved as above thanks to the fact that the homogeneous part of equations (4.10), (4.13) is exactly the same as of the equations (4.6), (4.9) above.

Part 2. Now we shall prove that system (4.20)-(4.22) has a unique solution. Using the Cauchy formula for (4.20) and the expressions $h_{z}(a)=-M^{\prime}\left(z^{*}\right) n^{*}(a), n^{*}(a)=e^{\int_{0}^{a} h_{n}(\eta) \mathrm{d} \eta} n^{*}(0)$, we obtain the following representation of the solution:

$$
n_{0}(a)=n^{*}(a)\left(\frac{n_{0}(0)}{n^{*}(0)}-a M^{\prime}(z) z_{0}-\int_{0}^{a} \frac{h_{0}(\eta)}{n^{*}(\eta)} \mathrm{d} \eta\right) .
$$

Then conditions (4.21)-(4.22) take the form of a linear system:

$$
\left(\begin{array}{cc}
1-\int_{0}^{A} \beta(a) \frac{n^{*}(a)}{n^{*}(0)} \mathrm{d} a & \int_{0}^{A} a \beta(a) n^{*}(a) M^{\prime}\left(z^{*}\right) \mathrm{d} a \\
-\int_{0}^{A} \gamma(a) \frac{n^{*}(a)}{n^{*}(0)} \mathrm{d} a & 1+\int_{0}^{A} a \gamma(a) n^{*}(a) M^{\prime}\left(z^{*}\right) \mathrm{d} a
\end{array}\right)\left(\begin{array}{c}
n_{0}(0) \\
z_{0}
\end{array}\right)=\left(\begin{array}{c}
-\int_{0}^{A} \beta(a) n^{*}(a) \int_{0}^{a} \frac{h_{0}(\eta)}{n^{*}(\eta)} \mathrm{d} \eta \mathrm{d} a \\
-\int_{0}^{A} \gamma(a) n^{*}(a) \int_{0}^{a} \frac{h_{0}(\eta)}{n^{*}(\eta)} \mathrm{d} \eta \mathrm{d} a
\end{array}\right) .
$$

The upper-left element of the matrix in the left-hand side is zero due to (6.5), while the other elements are nonzero due to $n^{*}(a) M^{\prime}\left(z^{*}\right)>0$. Thus the matrix is invertible and there is a unique solution of (4.20)-(4.22).

Acknowledgements. The authors are grateful to Sebastian Aniţa for his valuable comments. This research is financed by the Austrian Science Foundation (FWF) under grant No I 476-N13.

\section{References}

[1] L.-I. Aniţa, S. Aniţa, V. Arnăutu. Global behavior for an age-dependent population model with logistic term and periodic vital rates. Applied Mathematics and Computation, 206 (2008), No. 1, 368-379.

[2] S. Aniţa. Analysis and Control of Age-Dependent Population Dynamics, Mathematical Modelling Series. Springer, 2000.

[3] L.-I. Anița S. Anița, V. Arnăutu. Optimal harvesting for periodic age-dependent population dynamics with logistic term. Applied Mathematics and Computation, 215 (2009), 2701-2715.

[4] S. Aniţa, V. Arnăutu, R. Ştefănescu. Numerical optimal harvesting for a periodic age-structured population dynamics with logistic term. Numerical Functional Analysis and Optimization, 30 (2009), No. 3-4, 183-198.

[5] F.J. Aragon, A.L. Dontchev, M. Gaydu, M.H. Geoffroy, V.M. Veliov. Metric Regularity of Newton's Iteration. SIAM Journal on Control and Optimization, 49 (2011), No. 2, 339-362.

[6] C. W. Clark. Mathematical Bioeconomics: The Optimal Management of Renewable Resources, John Wiley, New York, 1976.

[7] F. Colonius, W. Kliemann. Infinite time optimal control and periodicity, Applied Mathematics and Optimization. 20 (1989), No. 1, 113-130.

[8] A.L. Dontchev, R.T. Rockafellar. Implicit Functions and Solution Mappings. Springer Mathematics Monographs, Springer, Dordrecht, 2009.

[9] A.L. Dontchev, V.M. Veliov. Metric Regularity under Approximations. Control \& Cybernetics, (2009), 38/4B, No. 4, 1283-1303.

[10] G. Feichtinger, G. Tragler, V.M. Veliov. Optimality conditions for age-structured control systems. J. Math. Anal. Appl., 288, (2003), No. 1, 47-68.

[11] R. F. Hartl, On the properness of one-dimensional periodic control problems. Systems \& Control Letters, 20 (1993), No. 5, 393-395.

[12] M. Iannelli. Mathematical theory of age-structured population dynamics, Applied mathematics monographs. C.N.R., Giardini editori e stampatori, Pisa, 1995.

[13] O. Tahvonen. Age-sructured optimization models in fisheries bioeconomics, Taylor and Francis, (2011), 140-173.

[14] H. Thieme, Mathematics in Population Biology, Mathematical Biology Series, Princeton University Press, 2003.

[15] G. F. Webb. Theory of Nonlinear Age-Dependent Population Dynamics. Marcel Dekker, New York, 1985. 